\title{
LOS BORDES DEL PROTECCIONISMO: LÍMITES Y RESTRICCIONES AL EJERCICIO DE LOS DERECHOS HUMANOS ${ }^{1}$
}

The edges of protectionism: limits and restrictions on the exercise of human rights

$$
\text { Roy GONZÁLEZ PADILLA }
$$

\section{Sumario:}

I. Introducción. II. Límites y restricciones: condiciones mínimas para su imposición. III. La SCJN y la interpretación de los criterios internacionales. IV. ¿La excepción a la regla o la regla de la excepción? V. Algunas notas conclusivas. VI. Bibliografía.

Resumen. En una reciente decisión tomada por la Suprema Corte de Justicia de la Nación, el Pleno resolvió que las restricciones a los derechos humanos expresamente contenidas en la Constitución deberán ser respetadas en todo momento sobre las disposiciones reguladas en los tratados internacionales firmados por México. En lo subsecuente, este documento presentará una discusión sobre la importancia que ha cobrado, en virtud de esta decisión jurisdiccional, la fuerza jurídica de las restricciones impuestas al ejercicio de los derechos humanos en el país.

Palabras clave: derechos humanos, límites, restricciones, protección.

Abstract. In a recent decision taken by the Supreme Court of Justice of Mexico, the Court stated that restrictions on human rights expressly contained in the country's constitution must be respected at all times over those human rights provisions regulated by international treaties signed by Mexico. Hereafter, this paper will present a discussion of the importance acquired, by virtue of this jurisdictional decision, the legal force of the restrictions imposed on the exercise of human rights in the country.

Keywords: human rights, limits, restrictions, protection.

\footnotetext{
${ }^{1}$ En la preparación de éste artículo de investigación resultaron ilustradoras las discusiones que sostuve con Gabriela Zaragoza sobre los temas aquí tratados, quien por lo general me hacía ver las imprecisiones de mi postura. De igual forma, los comentarios de los evaluadores anónimos abonaron a la versión que aquí se presenta. En cualquier caso, el texto definitivo es de mi entera responsabilidad.

${ }^{2}$ Candidato a Doctor en Ciencias Políticas y Sociales por la Universidad Nacional Autónoma de México. Profesor-investigador invitado en el Departamento de Estudios Políticos y de Gobierno de la División de Derecho, Política y Gobierno, Universidad de Guanajuato, Campus Guanajuato.
} 


\section{INTRODUCCIÓN}

Durante los meses de agosto y septiembre del 2013 el Pleno de la Suprema Corte de Justicia de la Nación (SCJN) llevó a cabo el análisis, discusión y resolución de un par de temas contenidos en la contradicción de tesis 293/2011 sometidos a su consideración. ${ }^{3}$

Las cuestiones que se discutieron en ambos temas giraron en torno a dos ejes principales: a) la fuerza jurídica de las restricciones impuestas al ejercicio de los derechos humanos reguladas en la Constitución frente a las disposiciones contenidas en el derecho internacional, y b) el carácter vinculante de la jurisprudencia emitida por la Corte Interamericana de Derechos Humanos (CIDH).

Sin negar el valor concomitante que comparten ambos criterios en el desarrollo del ejercicio de los derechos humanos en el ámbito interno, éste documento explorará el alcance de dicha resolución respecto a la primera de dichas cuestiones, esto es, se presentará una discusión sobre la importancia que ha cobrado, en virtud de esta decisión jurisdiccional, la fuerza jurídica de las restricciones impuestas al ejercicio de los derechos humanos en el país.

Según este orden de ideas este trabajo presentará a continuación, en primer lugar, una discusión sobre el asunto de la restricción legítima desde el derecho interno a los derechos humanos contenidos en instrumentos internacionales que permita guiar el análisis, en un segundo momento, de la decisión sostenida por la SCJN con motivo de la resolución de la contradicción de criterios sobre el particular antes citada; en tercer lugar, se confrontarán algunas apreciaciones doctrinales que se han vertido sobre esta posición jurisdiccional, y finalmente se concluirá con algunas notas sobre el alcance que la interpretación resuelta por la SCJN tiene para el entero del sistema de protección de derechos humanos en nuestro país.

\section{LÍMITES Y RESTRICCIONES: CONDICIONES MÍNIMAS PARA SU IMPOSICIÓN}

Es común a la doctrina y a la práctica jurisdiccional en la materia que los derechos humanos si bien no deben ser condicionados en cuanto a su ejercicio, sí se encuentran en cambio sujetos a ciertos límites y restricciones, ya sean estos explícitos o no.

Los derechos fundamentales son considerados como "atributos que jamás tienen alcance absoluto, pues si lo poseyeran se convertirían en prerrogativas típicas de un déspota que obra, con rasgos ilícitos o abusivos", ${ }_{4}^{4}$ de modo que su restricción se encuentra justificada en todo caso por exigencias propias de la vida social.

Así entonces su limitación no lleva necesariamente a restar el valor intrínseco que contienen o a mermar la relevancia que su regulación atañe al orden constitucional de un país, sino más bien obedece a que "la vida en sociedad exige que el ordenamiento jurídico también consagre limitaciones al ejercicio de todos los derechos humanos, en aras del bien común".5

En este sentido, por limitaciones a derechos fundamentales se entienden "aquellas restricciones al ejercicio de un determinado derecho básico, de manera tal que toda pretensión de ejercicio del atributo respectivo que vulnere los límites impuestos por las mismas es por

\footnotetext{
${ }^{3}$ El texto íntegro de la ejecutoria se encuentra disponible para su consulta en el siguiente vínculo: https://www. scjn.gob.mx/Transparencia/Epocas/Pleno/DecimaEpoca/293-2011-PL\%20CT\%2oEjecutoria.pdf.

${ }^{4}$ CEA, José Luis, Derecho Constitucional Chileno, t. II, Santiago de Chile, Ediciones Universidad Católica de Chile, 2002, p. 58.

${ }^{5}$ GUZMÁN, Jaime, Escritos personales, Santiago de Chile, Zig-Zag, 1992, p. 140.
} 
esencia antijurídica, y puede derivar para el titular infractor en las responsabilidades que para tal efecto prevea el ordenamiento jurídico positivo". 6

Ahora bien, por lo que hace a aquellos derechos fundamentales comprendidos en instrumentos internacionales, es de advertirse que los tratados que los contienen también regulan su posible restricción por parte de los Estados, facultad que sin embargo no es discrecional "sino que está limitada por el derecho internacional, que exige el cumplimiento de ciertas condiciones, cuya ausencia transforma la restricción en ilegítima y, por lo tanto, en violatoria de las obligaciones internacionales del Estado".

Es por ello que desde el derecho internacional se han formulado una serie de condiciones mínimas para poder llevar a cabo y justificar, desde el ámbito interno de los estados, la imposición de límites a derechos fundamentales [Cuadro 1].

\section{Cuadro 1. Condiciones mínimas para la imposición de límites a derechos fundamentales desde el derecho internacional}

\begin{tabular}{cl}
\hline CONDICIÓN & \multicolumn{1}{c}{ CARACTERÍSTICAS } \\
\hline & $\begin{array}{l}\text { Las restricciones serán aplicadas conforme a leyes que se dictaren por } \\
\text { razones de interés general y con el propósito para el cual han sido } \\
\text { establecidas, emanadas de los órganos legislativos constitucionalmente } \\
\text { previstos y democráticamente elegidos, y elaboradas según el procedimiento } \\
\text { previamente establecido. }\end{array}$ \\
$\begin{array}{c}\text { Entre tales propósitos legítimos específicos se cuentan el interés de la } \\
\text { Legitimidad de } \\
\text { los fines }\end{array}$ & $\begin{array}{l}\text { súblicas, la protección de las libertades de otros e incluso límites por razón } \\
\text { de interés social. }\end{array}$ \\
& $\begin{array}{l}\text { La condición de que dichas restricciones sean necesarias en una sociedad } \\
\text { democrática dependerá de que estén orientadas a satisfacer un interés }\end{array}$ \\
Deferencia & público imperativo, que de entre varias opciones para alcanzar ese objetivo \\
debe escogerse aquélla que restrinja en menor escala el derecho protegido y & sea proporcional al interés que la justifica. \\
\hline
\end{tabular}

Fuente: Elaboración propia a partir de las disposiciones contenidas en la CADH y

los criterios emitidos por la CIDH

En primer lugar se encuentra la condición de su establecimiento en una ley, según lo señalado en el artículo 30 de la Convención Americana sobre Derechos Humanos (CADH), en el que se señala que "las restricciones permitidas, de acuerdo con esta Convención, al goce y ejercicio de los derechos y libertades reconocidas en la misma, no pueden ser aplicadas sino conforme a leyes que se dictaren por razones de interés general y con el propósito para el cual han sido establecidas", condición que obedece a una necesidad de "salvaguarda a la posible arbitrariedad del gobierno, ya que la expresión 'ley' implica requisitos de forma y materiales, [...] salvaguarda tiene por objeto prevenir la imposición de restricciones por meros decretos ejecutivos".

Esta condición ha sido a su vez interpretada en el mismo sentido por la CIDH para quien "la palabra leyes en el artículo 30 de la Convención significa norma jurídica de carácter general, ceñida al bien común, emanada de los órganos legislativos constitucionalmente pre-

\footnotetext{
6 TÓRTORA, Hugo, "Las limitaciones a los derechos fundamentales", en Estudios Constitucionales, año 8, no. 2, 2010, pp. 169.

7 MEDINA, Cecilia y NASH, Claudio. Sistema Interamericano de Derechos Humanos: Introducción a sus Mecanismos de Protección, Santiago de Chile, Universidad de Chile, 2007, p. 32.

${ }^{8}$ MEDINA, Cecilia y NASH, Claudio, Op. Cit., pp. 32-33.
} 
vistos y democráticamente elegidos, y elaborada según el procedimiento establecido por las constituciones de los Estados partes para la formación de las leyes". 9

En segundo lugar tenemos la condición de que toda restricción que se pretenda imponer base su justificación en uno de los objetivos perseguidos en la propia CADH, ya sea que se trate de una restricción de carácter general o una de carácter específico para ciertos derechos. Entre tales propósitos legítimos específicos se cuentan el interés de la seguridad nacional, el orden público, la protección de la salud o la moral públicas, la protección de las libertades de otros e incluso límites por razón de interés social. ${ }^{10}$

En tercer lugar se enlista la condición de que dichas restricciones sean necesarias para el curso ordinario de una sociedad democrática, requisito que explícitamente hace referencia a los derechos de reunión pacífica, libertad de asociación y de movimiento contenidos en los artículos 15, 16 y 22 de la CADH.

Sobre esta expresión la $\mathrm{CIDH}$ ha considerado que la integran los siguientes elementos: a) que la «necesidad» del establecimiento de cualquier restricción dependerá de que estén orientadas a satisfacer un interés público imperativo; b) que de entre varias opciones para alcanzar ese objetivo deberá escogerse aquélla que restrinja en menor escala el derecho protegido; y c) que las restricciones deberán justificarse por la búsqueda de objetivos colectivos que, por su importancia, resulten preponderantes por sobre la necesidad social, es decir, sean proporcionales al interés que las justifican y ajustarse estrechamente al logro de esos legítimos objetivos. ${ }^{11}$

Según un criterio emitido por la SCJN, "ningún derecho fundamental es absoluto y en esa medida todos admiten restricciones, sin embargo, la regulación de éstas no puede ser arbitraria," ${ }^{12}$ de ahí que, de manera paralela a las condiciones establecidas por la CADH antes comentadas, desde el derecho interno también se han enunciado una serie de elementos mínimos para poder considerar válidas la imposición de restricciones a derechos fundamentales atendiendo a que, para la SCJN, las medidas emitidas por el legislador ordinario con el propósito de restringir derechos humanos sólo serán válidas si satisfacen cuando menos una serie de requisitos [Cuadro 2].

\footnotetext{
${ }^{9}$ CORTE INTERAMERICANA DE DERECHOS HUMANOS, La expresión "Leyes" en el Artículo 30 de la Convención Americana sobre Derechos Humanos, Opinión Consultiva OC-6/86, 9 de mayo de 1986, Serie A No. 6, párr. 10.

10 Artículos 12, 13, 15, 16, 22 y 32 de la CADH.

11 CORTE INTERAMERICANA DE DERECHOS HUMANOS, La colegiación obligatoria de periodistas, Opinión Consultiva OC-5/85, 13 de noviembre de 1985, Serie A No. 5, párr. 46.

12 TESIS 1a./J. 2/2012, Semanario Judicial de la Federación y su Gaceta, Décima Época, Libro V, febrero de 2012, p. 533 .
} 
Cuadro 2. Condiciones mínimas para la imposición de límites a derechos fundamentales desde el derecho interno

\begin{tabular}{cl}
\hline CONDICIÓN & \multicolumn{1}{c}{ CARACTERÍSTICAS } \\
\hline $\begin{array}{c}\text { Parámetro } \\
\text { constitucional }\end{array}$ & $\begin{array}{l}\text { El legislador ordinario sólo puede restringir o suspender el ejercicio de las } \\
\text { garantías individuales con objetivos que puedan enmarcarse dentro de las } \\
\text { previsiones de la Carta Magna. }\end{array}$ \\
& $\begin{array}{l}\text { El legislador deberá considerar si dicha restricción es el medio necesario } \\
\text { para proteger esos fines o intereses constitucionalmente amparados, lo que } \\
\text { significa que el fin buscado por el legislador no se pueda alcanzar } \\
\text { razonablemente por otros medios menos restrictivos de derechos } \\
\text { fundamentales. } \\
\text { fines }\end{array}$ \\
$\begin{array}{l}\text { La restricción legislativa se debe enmarcar dentro de las opciones de } \\
\text { tratamiento que pueden considerarse proporcionales, pues la persecución } \\
\text { de un objetivo constitucional no puede hacerse a costa de una afectación } \\
\text { innecesaria o desmedida a otros bienes y derechos constitucionalmente } \\
\text { protegidos. }\end{array}$ \\
\hline
\end{tabular}

Fuente: Elaboración propia a partir de los criterios emitidos por la SCJN.

En este sentido, el legislador debe determinar en cada caso si la restricción legislativa a un derecho fundamental es, en primer lugar, admisible dadas las previsiones constitucionales, ya que "el legislador ordinario sólo puede restringir o suspender el ejercicio de las garantías individuales con objetivos que puedan enmarcarse dentro de las previsiones de la Carta Magna", ${ }^{13}$ y por otro lado deberá considerar si dicha restricción es el medio necesario para proteger esos fines o intereses constitucionalmente amparados, esto es, "no basta que la restricción sea en términos amplios útil para la obtención de esos objetivos, sino que debe ser la idónea para su realización, lo que significa que el fin buscado por el legislador no se pueda alcanzar razonablemente por otros medios menos restrictivos de derechos fundamentales". ${ }^{14}$

Y por último el legislador ordinario deberá acreditar si la restricción legislativa se encuentra dentro de las opciones de tratamiento que pueden considerarse proporcionales, es decir, "la medida legislativa debe respetar una correspondencia entre la importancia del fin buscado por la ley, y los efectos perjudiciales que produce en otros derechos e intereses constitucionales, en el entendido de que la persecución de un objetivo constitucional no puede hacerse a costa de una afectación innecesaria o desmedida a otros bienes y derechos constitucionalmente protegidos" ${ }^{15}$

En suma, en ambos casos estamos ante la presencia de condiciones formales y materiales mínimas que tanto desde el derecho internacional como desde el ámbito interno de los estados se han formulado - a través de las interpretaciones emitidas desde su respectiva jurisdicción por la CIDH y la SCJN- para poder llevar a cabo y justificar la imposición de límites a derechos fundamentales.

Es de advertirse asimismo que dicha regulación se dirige a delinear parámetros mínimos para la imposición legítima de restricciones a derechos fundamentales en el marco de un tipo de situación general denominado «normalidad institucional», es decir, no se trata de aquellas suspensiones extraordinarias a derechos previstas por el orden constitucional en los

\footnotetext{
${ }^{13}$ Ídem.

${ }^{14}$ Ídem.

${ }^{15}$ Ídem.
} 
casos de invasión, perturbación grave de la paz pública o de cualquier otro que ponga a la sociedad en grave peligro o conflicto, ${ }^{16}$ sino de limitaciones ordinaras "que operan siempre, y que afectan el ejercicio de un derecho tanto bajo condiciones de normalidad constitucional, como bajo situaciones de excepción constitucional. Representan la regla general y se aplican en todo momento", ${ }^{17} \mathrm{y}$ se encuentran además expresamente previstas por el ordenamiento jurídico positivo de rango constitucional. ${ }^{18}$

\section{LA SCJN Y LA INTERPRETACIÓN DE LOS CRITERIOS INTERNACIONALES}

En el mes de junio de 2011 se publicó de manera oficial una reforma constitucional que se tradujo en una modificación sustantiva de la Carta Maga que, por un lado, delineó en su conjunto un nuevo paradigma en el constitucionalismo mexicano al introducir el concepto de los derechos humanos como eje central de la articulación normativa y, por el otro, incorporó como normas de máximo rango en el ordenamiento jurídico mexicano las disposiciones en materia de derechos de origen internacional. ${ }^{19}$

De entre las diversas dimensiones del impacto notable que dichas reformas han tenido en el ordenamiento jurídico del país cabe destacar el fortalecimiento del estatus jurídico de los tratados internacionales de derechos humanos, al haberse precisado su jerarquía de rango constitucional y estableciendo, entre otras cosas, que las normas relativas a los derechos fundamentales se interpretarán de conformidad con la Constitución y los propios tratados, ${ }^{20}$ lo que modificó, en consecuencia, los criterios sobre el valor constitucional de los tratados in-

\footnotetext{
${ }^{16}$ En este sentido, el artículo 29 de Constitución Política de los Estados Unidos Mexicanos es claro al señalar las causas de excepción, el procedimiento y los derechos que se encuentran excluidos de dicha suspensión. De igual forma esta facultad se encuentra regulada por el artículo 27 de la CADH. Un análisis teórico realizado a la luz de la reforma constitucional en materia de derechos humanos se encuentra en SALAZAR, Pedro, "Del estado de excepción a la suspensión constitucionalizada. Reflexiones sobre la reforma al artículo 29 de la Constitución mexicana", en La reforma constitucional de derechos humanos: un nuevo paradigma, CARBONELL, Miguel y SALAZAR, Pedro (coords.). México, UNAM, 2011, pp. 257-291.

17 TÓRTORA, Hugo, Op. Cit., p. 169.

${ }^{18}$ Otro tipo de limitaciones a derechos fundamentales son aquellas restricciones que nacen del respeto por los derechos fundamentales de los demás sujetos, circunstancia que impide a cada titular utilizar su derecho en perjuicio de otro (artículo 32.2 de la CADH), y aquellas de carácter u origen material o físico que tienen que ver con las reales posibilidades del Estado para responder a determinados derechos llamados "prestacionales" o de "segunda generación” (CEA, José Luis, Op. Cit., p. 63), restricciones que no son del interés del presente análisis. Asimismo cabe aclarar que en el sistema jurídico mexicano la competencia para imponer restricciones no solamente corresponde a la Carta Magna, sino también existen "restricciones indirectamente constitucionales” (ALEXY, Robert. Teoría de los Derechos Fundamentales. Madrid, Centro de Estudios Políticos y Constitucionales, 2002, p. 282) que se refieren a cláusulas de reserva explícitas en la propia norma fundamental que habilitan expresamente intervenciones de las legislaciones secundarias para generar restricciones a determinados derechos.

${ }^{19}$ CARBONELL, Miguel y SALAZAR, Pedro, Op. Cit., p. X.

${ }^{\circ}$ SALTALAMACCHIA, Natalia y COVARRUBIAS, Ana, "La dimensión internacional de la reforma de derechos humanos: antecedentes históricos”, en CARBONELL, Miguel y SALAZAR, Pedro, Op. Cit., p. 1. 
ternacionales en la materia en relación con una posición específica en la pirámide normativa para dirigirse hacia una "cláusula de interpretación conforme». ${ }^{21}$

De acuerdo con el análisis propuesto por Caballero ${ }^{22}$ este nuevo tratamiento constitucional trae una serie de ventajas, entre las que cabe destacar: a) la eliminación de la carga de divisiones y subdivisiones jerárquicas que se contenía en el antiguo artículo 133 constitucional previo a dicha reforma; b) el reconocimiento de la autonomía del derecho internacional y de los tratados que encuentran en sí mismos el fundamento de su vigencia, modificación e interpretación -situándose respecto del orden jurídico interno como ámbitos normativos distintos a los que se no se accede necesariamente a través del principio de jerarquía normativa, sino mediante el de distribución de competencias-; y c) la evolución de los criterios jurisprudenciales de los organismos a cargo de la interpretación del contenido esencial de los derechos fundamentales ${ }^{23}$ - quizá la más importante de dichas ventajas-.

Esta evolución de la función jurisdiccional que importa la complejidad del razonamiento judicial bajo los parámetros hermenéuticos antes comentados, ha traído como tarea adicional a la ordinaria de interpretar las leyes "la dificultad de trabajar con [nuevos] valores que están constitucionalizados y que requieren una tarea hermenéutica que sea capaz de aplicarlos a los casos concretos de forma justificada y razonable, dotándolos de esa manera de contenidos normativos concretos". ${ }^{24}$

Desde esta perspectiva teórica es posible analizar la contradicción de tesis 293/2011 resuelta por el Pleno de la SCJN a finales del 2013, tomándola como un caso paradigmático de esta nueva hermenéutica jurisdiccional en materia de derechos humanos.

En dicha contradicción de tesis se plantearon diversos asuntos relevantes sobre el denominado bloque de constitucionalidad, la supremacía constitucional, la jerarquía de normas de derechos humanos contenidas en tratados internacionales y sobre el carácter vinculante de precedentes de la CIDH.

En aquel año la controversia interpretativa suscitada por un par de Tribunales Colegiados de Circuito sobre la jerarquía de las normas sobre derechos humanos contenidas en tratados y su interpretación por parte de instancias internacionales fue previa a la reforma en la materia antes comentada, circunstancia que si bien entonces no ponía en el centro del análisis el recién reformado texto constitucional, no desmerecía empero la atención privilegiada por parte del más alto tribunal constitucional del país debido a la trascendencia que la definición

\footnotetext{
${ }^{21}$ En términos generales la llamada «cláusula de interpretación conforme» se puede entender como una "técnica hermenéutica por medio de la cual los derechos y libertados constitucionales son armonizados con los valores, principios y normas contenidos en los tratados internacionales sobre derechos humanos signados por los estados, así como por la jurisprudencia de los tribunales internacionales (y en ocasiones otras resoluciones y fuentes internacionales), para lograr su mayor eficacia y protección" (FERRER, Eduardo, "Interpretación conforme y control difuso de convencionalidad. el nuevo paradigma para el juez mexicano", en CARBONELL, Miguel y SALAZAR, Pedro, Op. Cit., p. 358).

${ }^{22}$ CABALLERO, José Luis, "La cláusula de interpretación conforme y el principio pro persona (artículo 10., segundo párrafo, de la Constitución)”, en CARBONELL, Miguel y SALAZAR, Pedro, Op. Cit., pp. 108-110.

${ }^{23}$ Este nuevo modelo de prácticas jurisprudenciales viene a constituir, en combinación con el surgimiento de novedosos textos constitucionales y desarrollos teóricos distintivos, el fenómeno normativo conocido como «neoconstitucionalismo» (CARBONELL, Miguel. Teoría del neoconstitucionalismo. Ensayos escogidos, Madrid, Trotta, 2007).

24 CARBONELL, Miguel, Op. Cit., p. 10.
} 
de los alcances del nuevo artículo primero constitucional tendría para el entero del sistema jurídico mexicano en lo subsecuente. ${ }^{25}$

Dicha contradicción de tesis versó fundamentalmente sobre dos cuestiones: en primer lugar, se sostuvieron criterios encontrados respecto a qué posición deberían ocupar los tratados internacionales en materia de derechos humanos en relación a la Carta Magna; y en segundo lugar, sobre qué tratamiento se le debería otorgar a la jurisprudencia emitida en la materia por parte de la CIDH.

Por lo que hace a los criterios sostenidos respecto al deber que tienen todas las autoridades de cumplir y hacer cumplir los tratados internacionales en materia de derechos humanos suscritos, a través del ejercicio del control de convencionalidad entre las normas jurídicas internas y las contenidas en documentos internacionales, ambos tribunales sostuvieron la pertinencia del control de convencionalidad desde el derecho interno y en consecuencia la SCJN resolvió que no existía un "punto de toque» entre ambas consideraciones respecto a dicho tema; sin embargo, respecto a la posición jerárquica de los tratados internacionales en materia de derechos humanos en relación con la Constitución y el carácter vinculante de la jurisprudencia en la materia emitida por la CIDH se presentaron primordialmente criterios discordantes [Cuadro 3].

Cuadro 3. Confrontación de criterios sostenidos en la contradicción de tesis 293/2011

\begin{tabular}{|c|c|c|}
\hline $\begin{array}{l}\text { TEMAS BASE DE LA } \\
\text { DISCUSIÓN }\end{array}$ & $\begin{array}{l}\text { 7MO. TRIBUNAL COLEGIADO EN } \\
\text { MATERIA CIVIL DEL 1ER. CIRCUITO }\end{array}$ & $\begin{array}{c}\text { 1ER. TRIBUNAL COLEGIADO EN } \\
\text { MATERIAS ADMVA. Y DE TRABAJO DEL } \\
\text { 11ER. CTO. }\end{array}$ \\
\hline $\begin{array}{l}\text { Posición jerárquica de } \\
\text { los tratados } \\
\text { internacionales en } \\
\text { materia de derechos } \\
\text { humanos en relación } \\
\text { con la Constitución }\end{array}$ & $\begin{array}{l}\text { Dichos tratados se encuentran por encima de } \\
\text { las leyes federales y por debajo de la } \\
\text { Constitución }\end{array}$ & $\begin{array}{l}\text { Cuando se trate de un conflicto que verse } \\
\text { sobre derechos humanos, los tratados o } \\
\text { convenciones internacionales suscritos por el } \\
\text { Estado Mexicano deben ubicarse } \\
\text { propiamente a nivel de la Constitución }\end{array}$ \\
\hline $\begin{array}{l}\text { Carácter de la } \\
\text { jurisprudencia en } \\
\text { materia de derechos } \\
\text { humanos emitida por } \\
\text { la Corte } \\
\text { Interamericana de } \\
\text { Derechos Humanos }\end{array}$ & $\begin{array}{l}\text { Es posible invocar la jurisprudencia de la } \\
\text { Corte Interamericana de Derechos Humanos } \\
\text { como criterio orientador cuando se trate de } \\
\text { la interpretación y cumplimiento de } \\
\text { disposiciones protectoras de los derechos } \\
\text { humanos }\end{array}$ & $\begin{array}{l}\text { Considera a la jurisprudencia internacional } \\
\text { en materia de derechos humanos como un } \\
\text { criterio obligatorio, el cual debe ser aplicado } \\
\text { por todas las autoridades con funciones } \\
\text { materialmente jurisdiccionales }\end{array}$ \\
\hline $\begin{array}{l}\text { Control de } \\
\text { convencionalidad }\end{array}$ & $\begin{array}{l}\text { Es necesario que los tribunales federales } \\
\text { ejerzan el denominado control de } \\
\text { convencionalidad }\end{array}$ & $\begin{array}{l}\text { Los tribunales del Estado mexicano no deben } \\
\text { limitarse a aplicar sólo las leyes locales, sino } \\
\text { también los tratados o convenciones } \\
\text { internacionales, lo cual obliga a ejercer el } \\
\text { control de convencionalidad entre las } \\
\text { normas jurídicas internas y las contenidas en } \\
\text { tratados internacionales }\end{array}$ \\
\hline
\end{tabular}

Fuente: Contradicción de tesis 293/2011.

\footnotetext{
${ }^{25}$ En lo que compete a los asuntos aquí tratados, la redacción actual del artículo $1^{\circ}$ constitucional expresa: "En los Estados Unidos Mexicanos todas las personas gozarán de los derechos humanos reconocidos en esta Constitución y en los tratados internacionales de los que el Estado Mexicano sea parte, así como de las garantías para su protección, cuyo ejercicio no podrá restringirse ni suspenderse, salvo en los casos y bajo las condiciones que esta Constitución establece. Las normas relativas a los derechos humanos se interpretarán de conformidad con esta Constitución y con los tratados internacionales de la materia favoreciendo en todo tiempo a las personas la protección más amplia. Todas las autoridades, en el ámbito de sus competencias, tienen la obligación de promover, respetar, proteger y garantizar los derechos humanos de conformidad con los principios de universalidad, interdependencia, indivisibilidad y progresividad. En consecuencia, el Estado deberá prevenir, investigar, sancionar y reparar las violaciones a los derechos humanos, en los términos que establezca la ley".
} 
Sentadas las bases de la contradicción en comento, el Pleno de la SCJN emitió una resolución definitiva que se sostuvo sobre tres ejes centrales: ${ }^{26}$

1. Los derechos humanos reconocidos en la Constitución y en los tratados internacionales adoptados por el estado mexicano conforman el parámetro de validez de toda la actuación pública.

2. Los posibles conflictos y antinomias entre derechos humanos reconocidos en la Constitución y en los tratados internacionales adoptados por el estado mexicano deben resolverse de acuerdo con el principio de la norma más favorable a la persona humana y no en términos jerárquicos; con una excepción: cuando en la Constitución haya una restricción expresa al ejercicio de los derechos humanos se deberá estar a lo que indica la norma constitucional.

3. La jurisprudencia de la CIDH tiene carácter vinculante - tanto la derivada de asuntos contra el estado mexicano como la producida en asuntos frente a los demás estados miembros-.

Por lo que respecta particularmente sobre la excepción a la regla sostenida por la SCJN en el sentido de respetar las restricciones constitucionales sobre las disposiciones contenidas en instrumentos internacionales sin atender el principio pro persona, la SCJN abandona en un primer momento el enfoque tradicional de «jerarquía de fuentes» - según el cual los tratados internacionales se encuentran jerárquicamente «por debajo» de la Constitución y «por encima» del resto de normas jurídicas que forman parte del entramado normativo mexicano- que durante varios años se mantuvo como el criterio interpretativo por excelencia, por considerar que dicho criterio de jerarquía resulta insatisfactorio para dar cuenta de lo ocurrido con las normas de derechos humanos previstas en tratados internacionales, cuyo tratamiento adquirió una nueva dimensión a raíz de las reformas constitucionales en la materia.

En lo esencial, la SCJN consideró que la nueva conformación del catálogo de derechos humanos no puede ser estudiada en términos de jerarquía en virtud de que la citada reforma constitucional modificó esencialmente el artículo $1^{\circ}$ al integrar un catálogo de derechos y no para distinguir o jerarquizar esas normas en atención a la fuente de la que provienen.

Sin embargo, según fue considerado por el Ministro José Ramón Cossío Díaz - quien para el caso emitió un voto particular en contra de la decisión tomada por la mayoría-, la decisión del Pleno de la SCJN presenta una evidente contradicción: por un lado, tratándose de la «regla general», la protección máxima a favor de la persona será el criterio a hermenéutico a considerar frente a la presencia de un conflicto de derechos humanos o antinomias entre ellos; pero en el caso de la «excepción a la regla», es decir, cuando se esté frente a un conflicto entre las restricciones expresamente reguladas en la constitución general del país y aquellas contenidas en disposiciones normativas internacionales, se deberá abandonar el

\footnotetext{
${ }^{26}$ SILVA, Fernando, "Derechos humanos y restricciones constitucionales: ¿reforma constitucional del futuro vs Interpretación constitucional del pasado? (comentario a la C.T. 293/2011 del Pleno de la SCJN)", en Revista Mexicana de Derecho Constitucional, no. 30, enero-junio 2014, pp. 251-272.
} 
principio pro homine ${ }^{27}$ y colocar al derecho interno sobre las normas de derecho internacional, retomando en consecuencia el principio de jerarquía constitucional.

\section{IV. ¿LA EXCEPCIÓN A LA REGLA O LA REGLA DE LA EXCEPCIÓN?}

A partir de la adopción de esta decisión jurisdiccional, diversos análisis doctrinarios han señalado una serie de deficiencias y transgresiones al paradigma de los derechos humanos que subrayan en lo general un vaciado del núcleo central de la reforma constitucional ya comentada, al haberse optado por un sistema que busca favorecer las restricciones constitucionales expresas en detrimento del principio pro homine que obliga a brindar la protección más amplia a la persona.

En esta línea argumentativa se encuentran algunas consideraciones como las de Báez ${ }^{28}$ para quien la interpretación de la SCJN "que ordena privilegiar las restricciones constitucionales por encima de los derechos humanos reconocidos en tratados internacionales" configura una «jurisprudencia de contrarreforma», en virtud de que "es contraria y vulnera el principio pro homine, ya que no atiende a la protección más amplia, sino a un criterio de jerarquía constitucional, actualmente superado a la luz del derecho internacional de los derechos humanos, se ignora por completo el mandato de atender, de acuerdo al caso, a la protección más amplia y a la restricción menor a los derechos humanos, para señalar un criterio fijo que privilegia las restricciones constitucionales, aun cuando en tos tratados internacionales se encuentre una protección mayor".

E inclusive para algunos otros como Labardini ${ }^{29}$ "si la propia enmienda constitucional estableció que debe aplicarse el principio pro persona para las normas de derechos humanos, entonces, debía aplicarse un criterio en extremo restringido para no lastimar o limitar los derechos humanos de que goza toda persona por ser persona; o de lo contrario, sería regresar al otorgamiento (o reconocimiento) de derechos en los términos expresos constitucionales y no porque los derechos humanos realmente sean de las personas".

Sin embargo, a pesar del aporte teórico que trae consigo la discusión de la posible vulneración de principios normativos que guían el paradigma de los derechos humanos, no hay que olvidar que, después de todo, los tratados internacionales en la materia van siempre acompañados de la posibilidad de la existencia de peculiaridades geográficas en la aplicación de las obligaciones en ellos contenidas, y son precisamente los tribunales encargados de su

\footnotetext{
${ }^{27}$ Dicho principio se constituye como "un criterio hermenéutico que informa todo el derecho internacional de los derechos humanos, en virtud del cual se debe acudir a la norma más amplia, o a la interpretación más extensiva, cuando se trata de reconocer derechos protegidos, e inversamente, a la norma o a la interpretación más restringida cuando se trata de establecer restricciones permanentes al ejercicio de los derechos o su suspensión extraordinaria" (PINTO, Mónica, "El principio pro homine. Criterios de hermenéutica y pautas para la regulación de los derechos humanos", en La aplicación de los tratados de derechos humanos por los tribunales locales, ABREGÚ, Martín y COURTIS, Christian (comps.). Argentina, Editorial del Puerto, 1997, pp. 163-171).

${ }^{28}$ BÁEZ, José Francisco, “Jurisprudencia de contrarreforma (CT: 293/2011)”, en REDHES, Revista de Derechos Humanos y Estudios Sociales, año VI, no. 11, enero-junio 2014, p. 65.

${ }^{29}$ LABARDINI, Rodrigo, "El (inexistente) derecho humano más que otro", en Boletín Mexicano de Derecho Comparado, nueva serie, año XLVII, no. 139, enero-abril 2014, p. 342.
} 
aplicación concreta quienes tienen la no sencilla tarea de reconciliar —o de justificar su falta de reconciliación - las diferencias normativas y morales dentro de cada región específica.

Según este orden de ideas es que de manera paralela se han venido desarrollando desde el ámbito internacional una serie de criterios interpretativos que conceden un cierto grado de «deferencia» a los estados parte de dichos tratados a efecto de que sean ellos mismos quienes regulen el contenido de los derechos fundamentales así como sus restricciones, y para distribuir el poder y los niveles de toma de decisiones entre autoridades domésticas y las cortes internacionales - criterios entre los que se encuentra la teoría de interpretación judicial internacional conocida como «margen de apreciación $»^{30}-$.

Esta noción "se funda en la capacidad que tiene la jurisdicción interna de interpretar los derechos humanos" - que se reconocen internacionalmente pero en muchos casos no su alcance-cuyos "límites se entrecruzan entre la noción de «razón de Estado" que le permite al Estado suspenderlos y restringirlos y, por otro lado, el control de esos límites por parte de las cortes regionales de derechos humanos que los racionalizan a través de interpretaciones extensivas de los derechos" ${ }^{31}$

Si bien esta teoría hermenéutica no ha sido citada explícitamente en los casos en los que México ha sido involucrado, ${ }^{32}$ la contradicción de tesis 293/2011 resuelta por la SCJN analizada es un ejemplo que pone en evidencia el uso de dicho método interpretativo por parte de un tribunal nacional con el fin de traducir y aplicar los tratados internacionales de derechos humanos de los cuales se es parte, haciendo uso de dicho "poder de deferencia» frente una circunstancia sobre la que no existe ningún tipo de consenso internacional - como lo son las posiciones que los altos tribunales nacionales fijan respecto a las restricciones a derechos fundamentales-, atendiendo además a que precisamente "la ausencia de consenso entre los Estados sobre temas sensibles en derechos humanos" ha llevado a que la CIDH justifique "una imposibilidad de definición en la interpretación y aplicación de algunos derechos y procedan al reconocimiento de un principio de deferencia hacia las autoridades nacionales". ${ }^{33}$

De ahí el desarrollo desde tribunales internacionales de una serie de «test doctrinales» con el ánimo de revisar la conducta de los estados parte en los procesos de restricción del alcance y la extensión de los derechos humanos [Cuadro 1], controles que de manera paralela han sido racionalizados y adecuados al contexto nacional a través de interpretaciones extensivas de dichos procesos [Cuadro 2].

\footnotetext{
${ }^{30} \mathrm{Cfr}$. ALVARADO, Paola Andrea y NÚÑEZ, Manuel, El margen de apreciación en el sistema americano de derechos humanos: proyecciones regionales y nacionales, México, UNAM, 2012; y LEGG, Andrew. The margin of appreciation in international human rights. Law deference and proportionality, United Kingdom, Oxford University Press, 2012.

${ }^{31}$ BARBOSA, Francisco, "El margen nacional de apreciación en el derecho internacional de los derechos humanos: entre el Estado de derecho y la sociedad democrática”, en El margen de apreciación en el sistema americano de derechos humanos: proyecciones regionales y nacionales, ALVARADO, Paola Andrea y NÚNEZ, Manuel, Op. Cit., pp. 56-57.

${ }^{32}$ PUENTE DE LA MORA, Gloria Margarita, "El Estado mexicano y la Corte Interamericana de los Derechos Humanos. Algunas consideraciones respecto al margen de apreciación en los casos contenciosos. Retos y perspectivas", en El margen de apreciación en el sistema americano de derechos humanos: proyecciones regionales y nacionales, ALVARADO, Paola Andrea y NÚÑEZ, Manuel, Op. Cit., pp. 285-317.

${ }^{33}$ BARBOSA, Francisco, Op. Cit., p. 74.
} 


\section{ALGUNAS NOTAS CONCLUSIVAS}

Es posible convenir junto con Contreras ${ }^{34}$ que si bien "la discreción nacional y la supervisión internacional son dos conceptos que estarán en permanente tensión en el trabajo de los tribunales regionales de derechos humanos", la interpretación jurisprudencial emitida por la SCJN aquí analizada muestra cómo la tensión concerniente a la restricción de derechos humanos ha tratado de ser resuelta mediante la distinción de tres niveles de condiciones mínimas en cada sistema —-desde el derecho internacional y desde el derecho interno-.

Tal como se ha visto, el primero de dichos niveles hace referencia al requisito de legalidad de las restricciones de los derechos: en ambos casos, tanto la CIDH como la SCJN han optado por una reserva de ley que habla de la previsibilidad y formalidad de las restricciones; en el segundo nivel ambos tribunales han considerado que toda restricción a derechos debe invocar un objetivo o fin legítimos; y en el último nivel, dentro del cual se presenta la mayor disimilitud de criterios, se acotan dichas restricciones atendiendo, para el primero de dichos tribunales, a la necesidad propia de una sociedad democrática, y para el segundo de ellos, a la proporcionalidad que en todo caso guarden.

En suma, si bien los estándares de discreción que operan en ambos órdenes jurídicos muestran convergencia en las formas y divergencias en lo sustantivo, la interpretación emitida por la SCJN respecto a la fuerza jurídica de las restricciones impuestas al ejercicio de los derechos humanos reguladas en la Constitución frente a las disposiciones contenidas en el derecho internacional, lejos de constituir "un claro ejemplo del abuso que se puede dar en la función interpretativa de la Jurisprudencia y sus efectos en los Derechos Humanos", ${ }^{35}$ o de haber configurado, en la más extrema de dichas visiones, una situación en donde las restricciones tienen "más fuerza que el derecho mismo pudiendo incluso el derecho humano no nacer en el sistema jurídico mexicano, o nacer deforme", ${ }^{66}$ dicho proceso hermenéutico da cuenta en cambio del espinado y sinuoso camino que la justicia nacional tiene que recorrer dentro del "universalismo de los derechos consagrados en las convenciones regionales sobre derechos humanos" para permitir "la posibilidad de explicar las voces disonantes que existen, respetando las limitaciones intrínsecas y extrínsecas en el margen nacional de apreciación", ${ }^{37}$ así como de la exigencia de limitaciones al ejercicio de todos los derechos humanos en aras del bien común que la vida en sociedad importa.

En concreto, este tipo de decisiones jurisdiccionales ponen en relieve la existencia de un proceso de racionalización del derecho internacional desde el ámbito interno que abona a la precaria potencialidad de la reforma constitucional en materia de derechos humanos ya citada ${ }^{38}$ pues precisamente dicho proceso racionalizador del ius commune habla de la imperiosa necesidad de contextualizar este derecho común a las realidades propias de cada región para que no sean más que derechos en papel y se conviertan en efectivos derechos en acción que se puedan ejercer, cierto es, dentro de límites legítimos que la razón obliga y atañen a la configuración de todo orden constitucional instituido.

\footnotetext{
${ }^{34}$ CONTRERAS, Pablo, "National discretion and international deference in the restriction of human rights: a comparison between the jurisprudence of the European and the Inter-American Court of Human Rights", en Northwestern Journal of International Human Rights, vol. XI, no. 1, 2012, p. 81.

35 BÁEZ, José Francisco, Op. Cit., p. 70.

${ }^{36}$ LABARDINI, Rodrigo, Op. Cit., p. 344.

37 BARBOSA, Francisco, Op. Cit., p. 73.

${ }^{38}$ VÁZQUEZ, Daniel, “Los límites de la reforma constitucional en materia de derechos humanos en México: por un poder político desconcentrado”, en Isonomía, no. 39, octubre 2013, pp.161-181.
} 
VI. FUENTES

BIBLIOGRÁFICAS

ALEXY, Robert, Teoría de los Derechos Fundamentales, Madrid, Centro de Estudios Políticos y Constitucionales, 2002.

ALVARADO, Paola Andrea y NÚNEZ, Manuel, El margen de apreciación en el sistema americano de derechos humanos: proyecciones regionales y nacionales, México, UNAM, 2012.

BÁEZ, José Francisco, “Jurisprudencia de contrarreforma (CT: 293/2011)", en REDHES, Revista de Derechos Humanos y Estudios Sociales, año VI, no. 11, enero-junio 2014, pp. 59-71.

BARBOSA, Francisco, "El margen nacional de apreciación en el derecho internacional de los derechos humanos: entre el Estado de derecho y la sociedad democrática”, en El margen de apreciación en el sistema americano de derechos humanos: proyecciones regionales y nacionales, ALVARADO, Paola Andrea y NÚÑEZ, Manuel (coords.). México, UNAM, 2012, pp. 31-82.

CABALLERO, José Luis, "La cláusula de interpretación conforme y el principio pro persona (artículo 10., segundo párrafo, de la Constitución)", en La reforma constitucional de derechos humanos: un nuevo paradigma, CARBONELL, Miguel y SALAZAR, Pedro (coords.). México, UNAM, 2011, pp. 103-133.

CARBONELL, Miguel, Teoría del neoconstitucionalismo. Ensayos escogidos, Madrid, Trotta, 2007.

CARBONELL, Miguel y SALAZAR, Pedro (coords.). La reforma constitucional de derechos humanos: un nuevo paradigma, México, UNAM, 2011.

CEA, José Luis, Derecho Constitucional Chileno, t. II, Santiago de Chile, Ediciones Universidad Católica de Chile, 2002.

CONTRERAS, Pablo, "National discretion and international deference in the restriction of human rights: a comparison between the jurisprudence of the European and the Inter-American Court of Human Rights", en Northwestern Journal of International Human Rights, vol. XI, no. 1, 2012.

CORTE INTERAMERICANA DE DERECHOS HUMANOS, La colegiación obligatoria de periodistas, Opinión Consultiva OC-5/85, 13 de noviembre de 1985, Serie A No. 5. sobre Derechos Humanos, Opinión Consultiva OC-6/86, 9 de mayo de 1986, Serie A No. 6.

FERRER, Eduardo, "Interpretación conforme y control difuso de convencionalidad. el nuevo paradigma para el juez mexicano", en La reforma constitucional de derechos humanos: un nuevo paradigma, CARBONELL, Miguel y SALAZAR, Pedro (coords.). México, UNAM, 2011, pp. 339-429. 
GUZMÁN, Jaime, Escritos personales, Santiago de Chile, Zig-Zag, 1992.

LABARDINI, Rodrigo, "El (inexistente) derecho humano más que otro", en Boletín Mexicano de Derecho Comparado, nueva serie, año XLVII, no. 139, enero-abril 2014, pp. 331-344.

LEGG, Andrew, The margin of appreciation in international human rights. Law deference and proportionality, United Kingdom, Oxford University Press, 2012.

MEDINA, Cecilia y NASH, Claudio, Sistema Interamericano de Derechos Humanos: Introducción a sus Mecanismos de Protección, Santiago de Chile, Universidad de Chile, 2007 .

PINTO, Mónica, "El principio pro homine. Criterios de hermenéutica y pautas para la regulación de los derechos humanos", en La aplicación de los tratados de derechos humanos por los tribunales locales, ABREGÚ, Martín y COURTIS, Christian (comps.). Argentina, Editorial del Puerto, 1997, pp. 163-171.

PUENTE DE LA MORA, Gloria Margarita. "El Estado mexicano y la Corte Interamericana de los Derechos Humanos. Algunas consideraciones respecto al margen de apreciación en los casos contenciosos. Retos y perspectivas", en El margen de apreciación en el sistema americano de derechos humanos: proyecciones regionales y nacionales, ALVARADO, Paola Andrea y NÚÑEZ, Manuel (coords.). México, UNAM, 2012, pp. 285-317.

SALAZAR, Pedro, "Del estado de excepción a la suspensión constitucionalizada. Reflexiones sobre la reforma al artículo 29 de la Constitución mexicana”, en La reforma constitucional de derechos humanos: un nuevo paradigma, CARBONELL, Miguel y SALAZAR, Pedro (coords.). México, UNAM, 2011, pp. 257-291.

SALTALAMACCHIA, Natalia y COVARRUBIAS, Ana, "La dimensión internacional de la reforma de derechos humanos: antecedentes históricos", en La reforma constitucional de derechos humanos: un nuevo paradigma, CARBONELL, Miguel y SALAZAR, Pedro (coords.). México, UNAM, 2011, pp. 1-38.

SILVA, Fernando, "Derechos humanos y restricciones constitucionales: ¿reforma constitucional del futuro vs Interpretación constitucional del pasado? (comentario a la C.T. 293/2011 del Pleno de la SCJN)", en Revista Mexicana de Derecho Constitucional, no. 30, enero-junio 2014, pp. 251-272.

TESIS 1a./J. 2/2012, Semanario Judicial de la Federación y su Gaceta, Décima Época, Libro $\mathrm{V}$, febrero de 2012, p. 533.

TÓRTORA, Hugo, "Las limitaciones a los derechos fundamentales", en Estudios Constitucionales, año 8, no. 2, 2010, pp. 167-200.

VÁZQUEZ, Daniel, "Los límites de la reforma constitucional en materia de derechos humanos en México: por un poder político desconcentrado”, en Isonomía, no. 39, octubre 2013, pp.161-181. 